\title{
Ancrage territorial et hybridation des savoir- faire au sein d'un système agroalimentaire localisé. Le cas des fromages corses
}

Territorial anchorage and hybridisation of know-how within a LAFS. The Corsican cheese

\section{Pascale Moity Maïzi et Rémi Bouche}

\section{OpenEdition}

\section{Journals}

Édition électronique

URL : http://journals.openedition.org/economierurale/2970

DOI : 10.4000/economierurale.2970

ISSN : 2105-2581

\section{Éditeur}

Société Française d'Économie Rurale (SFER)

Édition imprimée

Date de publication : 21 mars 2011

Pagination : 24-38

ISSN : 0013-0559

Référence électronique

Pascale Moity Maïzi et Rémi Bouche, « Ancrage territorial et hybridation des savoir-faire au sein d'un système agroalimentaire localisé. Le cas des fromages corses », Économie rurale [En ligne], 322 | marsavril 2011, mis en ligne le 30 mars 2013, consulté le 19 avril 2019. URL : http://

journals.openedition.org/economierurale/2970; DOI : 10.4000/economierurale.2970 


\section{Ancrage territorial et hybridation des savoir-faire au sein d'un système agroalimentaire localisé Le cas des fromages corses}

Pascale MOITY MAÏZI •UMR Innovation, SupAgro, Montpellier, maizi@supagro.inra.fr Rémi BOUCHE † •INRA, Laboratoire de recherches sur le développement de l'élevage, Corte

\section{Contexte et problématique}

D ans l'actualité des politiques de développement qui se concentrent sur les conditions d'une durabilité économique, sociale et écologique des productions humaines, où le territoire semble devenir un repère incontournable, la question de l'ancrage d'un produit à son terroir conduit la recherche à s'interroger : en quoi des produits locaux sont-ils susceptibles de constituer des ressources activables par des collectifs humains au point de devenir aux yeux de tous des objets et moyens pertinents pour définir des Systèmes agroalimentaires localisés (SYAL) ? Cette question repose sur une première hypothèse implicite : un objet (produit, technique) est porteur et révélateur d'identités ; bien plus, il peut être emblématique d'un SYAL, c'est-à-dire d'une forme historique et spatialisée d'organisation agroalimentaire qui permet aux acteurs, qui l'appréhendent comme une ressource liée à un territoire, de construire un projet collectif de patrimoine, combinant un milieu de référence, des acteurs avec leurs identités et leurs réseaux, des savoirfaire ${ }^{1}$. Une seconde hypothèse en découle : ce projet prend forme dans un processus volontariste avec tout ce qu'il comporte comme épreuves de force et de légitimité où les débats, portant sur les spécificités ou les identités dont un produit serait porteur, interrogent nécessairement les savoir-faire dont

1. Les termes de ce projet permettent de circonscrire et de définir un SYAL. Voir Muchnik et de Sainte Marie (2010), p. 13. il est issu. C'est ainsi que les savoirs et les savoir-faire forment une composante essentielle de l'identification collective du lien à une origine (Bérard et Marchenay, 2004).

\section{Les éléments constitutifs d'un SYAL fromager corse}

En Corse, l'organisation agropastorale s'est développée historiquement en combinant une double transhumance des troupeaux vers les plaines littorales l'hiver et l'été sur de hauts plateaux d'estive. Des étapes intermédiaires d'alimentation des troupeaux, au printemps et à l'automne, aux alentours des villages en piémont permettaient une utilisation optimale des ressources fourragères. Pour accomplir ces transhumances loin des villages, les bergers s'organisaient en « compagnies » sur la base de l'entraide et de l'interconnaissance permettant un déploiement en réseaux et une gestion du cheptel sur de très vastes territoires. Les longs déplacements saisonniers ont par ailleurs conduit à une spécialisation des tâches liées à l'élevage conférant aux hommes le gardiennage, l'alimentation et la traite du bétail avec une première transformation du lait, permettant de stabiliser les protéines en un caillé transportable ; aux femmes revenait l'affinage. Les évolutions du siècle dernier, notamment l'arrivée d'industries transformatrices de lait et les phénomènes de déprise agricole (guerre, industrialisation) ont entamé le déclin de cette organisation pastorale longtemps indissociable de la société insulaire. Mais celle-ci conserve la mémoire de cet ancrage culturel au territoire, repérable dans l'actualité des 
pratiques et des réseaux sociotechniques autour des élevages.

Le fromage corse est issu de traditions séculaires d'élaboration spécifiques dont personne ne conteste la légitimité. Aujourd'hui, en l'absence de protection et face à l'engouement d'un marché tiré par la grande distribution (nationale et européenne), par le développement d'une consommation touristique estivale, ce fromage perçu comme un produit ancré au territoire historique des systèmes agro-pastoraux corses, semble mis en péril soit par des contrefaçons produites à l'extérieur de l'île, soit par l'offre industrielle locale de fromages élaborés à partir de lait importé. Le risque de contrefaçons est anxiogène pour les éleveurs et les transformateurs fermiers ${ }^{2}$. Il interpelle la société civile dans un contexte social parfois tendu' L'interprofession laitière ${ }^{4}$ tente donc de mettre en place un ou des signes officiels de qualité qui permettraient de reconnaître et de protéger les fromages corses. Mais les négociations entre acteurs s'avèrent difficiles : d'une part pour le choix d'un signe parmi les différents scénarios de qualification possibles, d'autre part pour formaliser des critères communs de ce que devrait être le fromage corse. Existe-t-il, en effet, un ou des types de fromage local ? Quelles sont les zones de productions actuelles ? Qui doit profiter de la rente supposée d'un signe officiel de qualité et qui doit en être exclu ?

Cette démarche de réflexion collective est en fait confrontée à la difficulté de qualifier (et donc de reconnaître) des ressources physiques spécifiques (races locales, alimentation, lait) en interaction dans des systèmes d'élevage pilotés par des pratiques et des

2. Cette anxiété s'exprime dans le film réalisé en mars 2007 par des étudiants de SupAgro-Montpellier, option VALOR (Moity Maïzi, Paquin, 2007). 3. La manchette du journal Corse Matin en juillet 2007 titre sur les menaces d'un mouvement séparatiste aux importateurs de lait.

4. L'interprofession laitière, institution reconnue par l'État regroupe trois collèges : industriel, apporteur, transformateur fermier. savoir-faire (d'élevage et de transformation du lait) qui sont en constante évolution. Les débats butent d'une part sur la difficulté à prendre en compte tous les attributs possibles pour définir officiellement un produit «typique » emblématique d'un SYAL, d'autre part sur le caractère politique des outils d'identification de la (ou des) ressource(s). Au-delà du choix consensuel sur des critères communs qui objectiveraient les techniques d'élevage et de transformation laitière, c'est la mise en œuvre de ces indicateurs par des collectifs diversifiés, dans une démarche de qualification qui paraît complexe : chacun mesure en effet les conséquences économiques, sociales et éthiques d'un acte collectif de fixation officiel d'accords et de critères sur un standard donné, qui peuvent déterminer l'appartenance ou l'exclusion d'un éleveur.

\section{Positionnements de la recherche}

Les travaux sur cette difficulté à construire et stabiliser une démarche officielle de certification sont nombreux. Notre position c'est d'abord de souligner ici l'originalité d'une situation où s'affrontent différents types d'acteurs et de métiers : des dissymétries se révèlent entre divers cadres cognitifs et sociaux, traduites dans des techniques différenciées que le langage a parfois du mal à objectiver dans des cadres formels. Nous évoquerons donc les dimensions particulières de ces ressources cognitives incorporées dans les actes techniques d'élevage et de transformation laitière, en distinguant celles liées à l'environnement (écosystème naturel et culturel) de celles qui se rattachent à des univers professionnels exogènes. Nous soulignerons par là le caractère complexe et évolutif de ces ressources que chacun réclame aujourd'hui comme étant traditionnelles dans un processus de création collective de patrimoine : ancré dans la modernité de nos économies, celui-ci est le produit d'une intention nécessairement collective qui interroge, d'une part la valeur des savoir-faire investis d'une dimension 
traditionnelle et, d'autre part, l'arbitraire évident d'une construction sociale qui se voudrait intégrative de la diversité alors même qu'elle semble devoir exclure.

Dans un contexte politique où le Savoir est envisagé comme la «dimension principale $d u$ processus de production ainsi que la condition première de son expansion » (Stehr, 2000), nous considérons les savoir-faire mis en débat dans ces démarches comme un «tout anthropologique » (Morin, 1973) combinant informations, connaissances, expériences, apprentissages et liens sociaux. Notre analyse des savoir-faire privilégie d'une part l'examen des articulations singulières entre registres techniques, relationnels et cognitifs développées par des éleveurs pour se maintenir dans un milieu complexe (élevage extensif) ; d'autre part une réflexion sur l'ancrage territorial, entendu ici comme conception nouvelle de la transmission intergénérationnelle, proche en cela de la notion de patrimoine (terme que nous intégrons alors comme autre indicateur d'un SYAL ${ }^{5}$ ).

Pour notre recherche, différentes méthodes issues de l'ethnologie et de l'ergonomie ont été mobilisées, permettant d'appréhender ensemble les registres techniques, relationnels et cognitifs constitutifs des savoir-faire :

- Quand l'activité est observable, la méthode de l'auto confrontation empruntée à la théorie du cours d'action (Theureau, 1992) permet, après enregistrement vidéo de l'acte technique, de revenir avec un opérateur sur son activité.

- Lorsque l'activité n'est pas directement observable (activité décalée dans le temps ou l'espace), la méthode de l'entretien d'explicitation (Vermersch, 2010) permet à l'opérateur de proposer un récit de ses pratiques et connaissances.

- Pour ces deux méthodes, des enregistrements sonores et vidéo ont permis de réaliser des confrontations croisées avec des experts

5. Ce «patrimoine » revêt une dimension forte dans un SYAL dès lors qu'il est revendiqué, porté par des acteurs locaux. (technologues fromagers, conseillers agricoles) ou des tiers (par exemple, confrontations entre éleveurs).

Ces méthodes ont été intégrées dans un dispositif d'enquêtes déployé sur l'ensemble du territoire corse. Nous avons rencontré des éleveurs ovins, caprins, des artisans et industriels laitiers, fournissant un corpus de plus de 150 heures d'enregistrements.

\section{Le savoir-faire Une ressource pas comme les autres}

Un aliment est appréhendé par ses fonctions nutritives (composantes physiques, organoleptiques) et par la matière première dont il est issu lui donnant une identité propre. Depuis longtemps, la certification des produits liés à une origine associe à ces premiers critères une caractérisation du système de production ainsi que les dimensions cognitives et historiques incorporées au produit fini, susceptibles d'ancrer un produit à un territoire de production et d'origine (Bérard et Marchenay, 2007). Cette procédure soulève toutefois des questions portant sur les méthodes et critères d'identification des systèmes de connaissances incorporés au produit d'une part, de leur protection d'autre part. Les savoir-faire ne forment pas un capital invisible, immatériel et immuable ; ils sont une composante à part entière de toute ressource matérielle humaine et plus encore que les composantes physiques ou biologiques, ils sont soumis à des interactions et tensions entre l'individu et le collectif, le local et le global, l'intentionnel et le subi, l'être et l'avoir été (expérience), dans un contexte en évolution constante lui aussi.

La valeur heuristique du concept de savoir-faire, dont les définitions ne sont de fait jamais stabilisées, nous permet de le mobiliser ou d'en appréhender les traductions sociales ou techniques selon différents points de vue théoriques. Deforge (1991) le définit par exemple comme la capacité de réussir une activité par un enga- 
gement personnel. Plus récemment MoityMaïzi et Muchnick (2005), Sigaut (2006) rejoignant Chevallier et Chiva (1991) s'accordent pour appréhender le savoir-faire comme "l'ensemble des compétences acquises, incorporées, transmises, qui se manifestent dans l'acte technique et qui supposent la mobilisation de nombreuses connaissances et représentations ». Les savoir-faire sont ainsi «perceptibles dans les attitudes physiques des individus comme dans leur capacité à juger, à prévoir et à maîtriser un processus technique. Par là, ils ne peuvent être réduits ni à un savoir ni à une technique » (op. cit.). Ils renverraient à une capacité de "voir en même temps devant et derrière », c'est-à-dire à se référer à l'expérience passée tout en anticipant une situation future. Cette perspective permet d'associer le savoir-faire à la métis grec décrite par Détienne et Vernant (1974), donnant à l'artisan qui la détient la capacité de s'adapter à des situations changeantes. Cette intelligence combine selon ces auteurs, "le flair, la sagacité, la prévision, la souplesse d'esprit, la feinte, la débrouillardise, l'attention vigilante, le sens de l'opportunité, des habiletés diverses " avec "une expérience longuement acquise » qui souligne, comme le note de son côté Schwint (2002), l'importance des temporalités et des apprentissages dans une construction cognitive, s'insérant entre théorie et pratique, savoir et agir, logos et praxis. Le territoire apparaît alors comme une traduction matérielle possible de ce double rapport au temps et à l'espace fondateur d'une connaissance humaine, qui en fait un vrai « laboratoire cognitif » (Moity-Maïzi et Muchnick, 2005).

Si la temporalité des savoir-faire est essentielle pour une démarche collective de qualification, comment peut-on alors repérer et décrire des savoir-faire revendiqués comme étant traditionnels par certains acteurs de la relance des fromages corses alors qu'ils sont nécessairement marqués d'histoires mêlées et d'hybridations successives ? Quels seraient les signes ou critères, tant techniques que symboliques, qui permettraient de les opposer à des savoirfaire qui seraient alors moins traditionnels ? Comment peut-on ensuite les formaliser ? Pour répondre à cette double interrogation, nous ferons l'hypothèse que le style technique telle la « griffe » d'un grand couturier, constitue l'élément clé de cette distinction : le style est en effet signe, support presque indicible de l'identité (individuelle ou collective) créative en même temps que prisonnière de normes (Moity Maïzi, 2009) ; il est support d'intégration en même temps que d'exclusion, inscrivant l'individu dans l'altérité. Il transcende par ailleurs l'opposition tradition/modernité par les significations et formes de reconnaissance qu'il véhicule. À partir de là « voir » les savoirfaire est aussi important que de les « recenser » dans un récit normatif. Identifier des savoir-faire conçus comme traditionnels repose donc sur l'observation et la mise en question de ces formes distinctives qui permettent d'opérer une différenciation dans chacune des productions matérielles d'un collectif. L'observation de la technique dans ses diverses dimensions opératoires, relationnelles, cognitives et stylistiques, est donc incontournable pour l'identification et pour la formalisation de savoir-faire. Mais la prudence éthique et méthodologique relative au recueil des savoir-faire nous amène par ailleurs à garder en conscience le paradoxe qui existe entre la nécessité de les « encoder » à travers cette formalisation, pour les rendre transmissibles aux générations futures et le fait que cet encodage augmente parallèlement le risque de contrefaçon ou de détournement. Enfin, en cherchant à caractériser ces savoir-faire dans un collectif donné, c'est bien aussi la société qu'il s'agit d'appréhender : avec le fait linguistique, le fait technique s'impose comme une des portes ouvrant directement sur la société (Guillet Escuret, 2003) ; le fait technique est ainsi heuristique en même 
temps que processus fait d'artefacts observables (Latour et Lemonnier, 1994). Il est le résultat historique de conditions naturelles, bio-physiques ou chimiques mais aussi de compromis, d'ajustements, de jugements. Plus précisément encore, il «se définit par la médiation des rapports entre les hommes d'une part, entre les hommes, les choses et les bêtes d'autres part»(Latour, 1993). Les connaissances, valeurs et symboles qui le structurent forment ainsi le cœur de toute investigation sur des activités matérielles et leurs produits.

Historiquement, les faits techniques sont ces multiples objets et actes (procédés, gestes) que l'histoire et l'anthropologie ont pu régulièrement inventorier et sérier pour établir notamment de grandes classifications des cultures matérielles. La critique de ce type de démarche bien résumée par Martinelli (1993) aboutit à l'explicitation d'une notion déjà centrale dans l'œuvre de Leroi Gourhan (1965), celle de tendance technique : «À partir d'une fonction générale et générique, des fonctions secondaires s'enchaînent et s'articulent à chaque degré du fait, révélatrices des choix et des contraintes qui règlent l'itinéraire de la tendance à travers le milieu technique et culturel » (Martinelli, 1993). La tendance technique se réalise à travers des choix qui font la diversité des faits techniques autour d'une même fonction : il y a ainsi plusieurs façons de faire pour regrouper un troupeau ; et si certains outils de fabrication fromagère répondent bien à une même catégorie de fonction, ils sont aussi façonnés, ajustés, stylisés, selon des principes propres à chaque groupe.

La tendance se concrétise donc aussi par « une stylisation» (Martinelli, 2005) qui touche les objets tout comme les gestes et produits d'une opération technique. L'intentionnalité qui préside à ces choix et qui fonde la diversité des actes techniques ne se réduit pas à l'anticipation ni à l'attente d'un résultat matériel précis; elle vise aussi des objectifs sociaux, symboliques ou poli- tiques, inscrits dans une gamme de possibles où distinction sociale et conformisme par rapport à un collectif de référence se mêlent et s'affrontent. On comprend mieux alors pourquoi d'un groupe à l'autre, d'une culture à une autre, les objets captent différemment l'attention d'un opérateur : ainsi par exemple, un éleveur face à son troupeau, et selon ses intentions, se concentre en priorité sur la santé de son cheptel, sur sa pâture ou sur la traite du lait.

\section{Le savoir élever au cœur de l'ancrage}

Dans les systèmes d'élevage traditionnels en Corse (Ravis-Giordanni, 1983), les éleveurs cherchent toujours à s'adapter au milieu et à gérer les incertitudes sur des temps longs. Ces systèmes pastoraux font appel à un usage intensif du travail humain qualifié difficile à formaliser : le pilotage des troupeaux sur de vastes territoires, la reconnaissance des animaux par certains signes ou par des formes de communication ne relevant pas nécessairement du langage courant, reposent sur des compétences d'adaptation construites dans et mobilisées par des réseaux socio-techniques complexes (Bouche, 2008). L'éleveur pastoral est en effet contraint de concevoir sa production comme une régulation des aléas du milieu qui interviennent :

- quotidiennement au gré des intempéries météorologiques;

- sur des fluctuations saisonnières combinant des périodes de raréfaction des ressources en hiver et en été avec des périodes d'abondance ;

- sur des variations interannuelles où se succèdent des années aux profils totalement différents. Les pratiques ne visent donc pas à maximiser l'expression du potentiel productif de l'animal en annulant les effets du milieu mais à combiner des performances individuelles dans l'entité «troupeau piloté ». C'est là que se construisent les éléments d'une compétence, caractérisée 
par la capacité d'établir des connexions complexes entre un système et son milieu ${ }^{6}$.

Ainsi, alors que schématiquement, les systèmes intensifs sécurisent et nivellent leurs dépendances aux aléas du milieu naturel en augmentant les niveaux d'intrant (alimentation fournie par exemple), et qu'à l'opposé les systèmes extensifs maximisent les surfaces et têtes de bétail au détriment du rendement, les systèmes pastoraux corses reposent sur des savoir-faire d'expériences permettant de se maintenir dans un milieu à forte incertitude. Cette complexité touche les dimensions internes au système (éleveur, troupeau, territoire) mais aussi les liens sociaux capables de le prendre en charge à différents niveaux d'organisation et dans des contextes variables.

Historiquement, le pilotage pastoral sur de grands espaces (parfois $>300$ ha) par exemple, a permis aux éleveurs de développer des compétences spécifiques relatives à leurs animaux comme aux lieux sur lesquels ils les nourrissent, induisant une série d'exigences socio-techniques. D'une part, le troupeau doit révéler, outre une grande adaptation au prélèvement de nourriture en milieu difficile (agilité, résistance, rusticité), des qualités d'ordre social (grégarisme, existence de meneuses dociles). D'autre part, il doit être repérable à distance (diversité des couleurs). Ces qualités sont anticipées, perçues puis maintenues par l'éleveur à travers ses pratiques de sélection animale. Par ailleurs, l'éleveur doit acquérir une connaissance fine des comportements animaux afin de pouvoir anticiper les réactions de chacun face aux aléas puis de réagir. Ces connaissances se construisent de manière dynamique au fil des générations dans une histoire pastorale et sociale organisant et régulant avec précision les formes et objets de transmissions intra et intergénérationnelles.

6. La théorie de la complexité énoncée par Wagensberg (1997) qui pose : "La complexité d'un système, moins sa capacité d'anticipation vis-à-vis de son milieu, est égale à l'incertitude du milieu moins sa sensibilité vis-à-vis du système. »
Si les bergers pilotent ainsi leurs troupeaux selon des scénarios complexes (par la diversité des éléments pris en compte par chaque individu), nous constatons qu'ils mobilisent aussi la présence d'autres usagers du territoire, envisagés comme des vecteurs d'informations ponctuelles (localisation d'un animal, état d'une ressource ou d'un chemin, activité d'un confrère éloigné etc.). L'élevage pastoral relève ainsi d'un pilotage distribué, fondé par une entraide informelle et indispensable, que nous proposons de nommer écholocation pastorale ${ }^{7}$ à l'aide d'un codage spécifique (marque aux oreilles, couleur des animaux) et d'une connaissance approfondie de la toponymie du territoire, l'éleveur peut retrouver un animal égaré ou connaître l'état d'une pâture distante par le biais de relais sociaux d'information. L'écholocation repose sur l'existence d'un réseau localisé d'usagers directement mobilisables et d'un vocabulaire partagé : piloter un troupeau en système extensif exige de mettre en œuvre des interactions particulières pour se connecter aux réseaux pastoraux et capturer ou émettre une information utile. Ces compétences demeurent aujourd'hui, mais le téléphone portable comme nouveau média de communication s'y substitue progressivement (Bouche, 2008).

Plus que la maximisation des performances, l'éleveur recherche ici une maîtrise du système dans sa globalité. L'homogénéité d'un troupeau par exemple se conçoit dès la mise bas. Outre la sélection du bon renouvellement $(20 \%)$, le suivi des mises bas permet un travail d'identification des cabris et d'appariement avec leurs mères. Il implique une présence et une attention constante pour s'assurer et mémoriser les filiations : «Moi si je ne vois pas la mise bas, je ne garde pas le cabri car il a pu être adopté. » Les éleveurs à la tête de petits effectifs avaient des pratiques similaires (par observation du troupeau durant le gardiennage quotidien) pour

7. Par analogie au système de pilotage des chauvessouris. 
mémoriser ${ }^{8}$ les couples mère-cabri. Actuellement, contraints d'augmenter l'effectif de leurs troupeaux ${ }^{9}$ pour raisons économiques, ils doivent adapter les artefacts cognitifs et organisationnels leur permettant de constituer un cheptel productif, où l'identification individuelle réglementaire (tip-tag) paraît fort éloignée de ce travail de repérage et de mémorisation. La reconnaissance de la flore susceptible de nourrir, de soigner le cheptel ou bien de nuire aux fromages est aussi un élément de maîtrise du système : telle pâture est potentiellement défavorable à la production laitière en volume « si je conduis demain les bêtes sous cette crête, elles vont chuter de 20 litres de lait demain », ou en qualité « si elles passent sous les oliviers, le fromage va couler ». Ces savoirs véhiculés dans des réseaux socio-techniques denses sont même ritualisés dans l'ensemble de la société par des adages, proverbes ou calendriers événementiels qui s'y réfèrent en permanence.

\section{Savoir fromager Un ancrage délégué}

Dans un tel système, les actes techniques et les priorités cognitives sont focalisés sur l'élevage de l'animal. L'activité de transformation du lait en fromage et sa commercialisation sont d'ailleurs éventuellement déléguées à un tiers.

Si l'éleveur est aussi transformateur, il peut encore établir des priorités entre tâches indispensables au maintien d'une cohérence globale de son système. Il n'est pas impossible par exemple de voir un éleveur reporter la fabrication fromagère pour partir chercher un animal perdu.

8. Certains parlent d'un don : "Le don si tu l'as pas à la mise bas, quand tous les cabris tombent en même temps, tu es perdu. " D'autres évoquent la nécessité absolue "d'être né dedans » pour apprendre ce métier.

9. L'effectif moyen aujourd'hui se situe autour de 180 têtes, avec toutefois des troupeaux de 500 à 600 têtes.
Même s'il existe de réels savoir-faire fromagers, la chaîne opératoire visant à stabiliser de façon rapide les protéines du lait pour leur conservation pouvait être à tout instant interrompue et déléguée à un tiers. L'affinage lui-même, pensé comme secondaire par le berger, au profit d'une surveillance du cheptel, du territoire et des informations susceptibles d'en faciliter la maîtrise, a toujours été confié aux femmes ${ }^{10}$ ou aux vieillards. Nos recherches historiques montrent que cette hiérarchisation des tâches a été renforcée au siècle dernier par la spécialisation professionnelle de certains éleveurs devenus livreurs de lait aux laiteries de Roquefort et déléguant définitivement à ces entreprises les opérations de transformation et de commercialisation.

\section{Le savoir-faire archétype de l'ancrage territorial}

Toutes ces observations empiriques dans la durée nous montrent que le savoir-faire est bien rattaché à des exigences d'anticipation, de communication et de coordination, qui l'inscrivent dans l'action collective. Celle-ci s'avère par ailleurs indispensable à la viabilité des pratiques individuelles dans un système pastoral extensif et contribue à son ancrage territorial.

Dans sa composante traditionnelle, le pastoralisme corse peut être analysé comme un archétype des savoir-faire collectifs ancrés à un territoire (SFCAT) (Bouche et al., 2009) qui articule sans les opposer dans un même ensemble :

- des compétences «phénotypiques » mises à l'épreuve quotidiennement (savoir anticiper, se mettre en relation, par exemple) ;

- les attributs intangibles de ces compétences qui les rendent reproductibles localement d'une génération à l'autre mais non géné-

10. Les savoir-faire associés à l'élaboration du goût sont dans ce contexte transmis dans un registre domestique de transmission féminin comme les actes de couture ou de cuisine. 


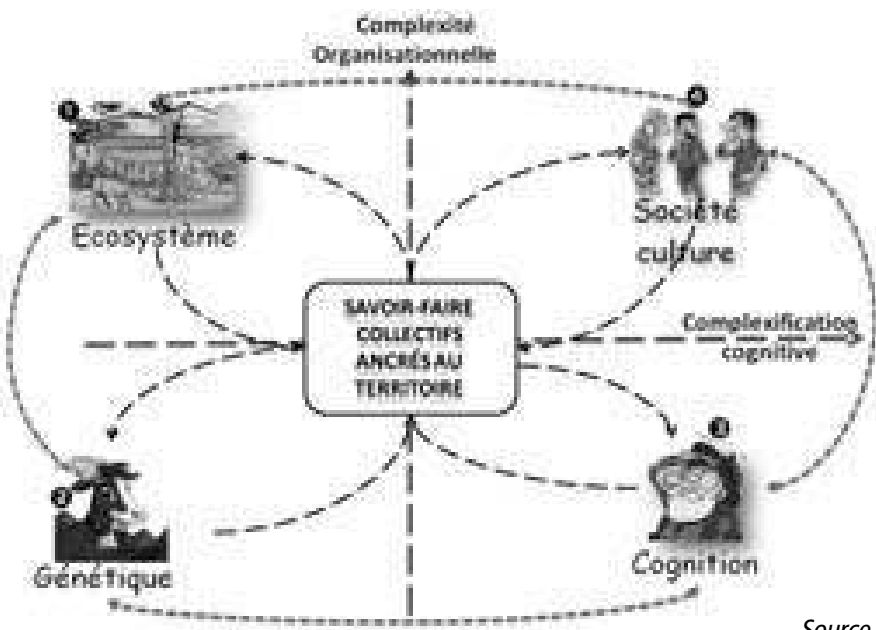

Source : Rémi Bouche

ralisables et qui en garantissent l'origine en même temps que l'originalité.

Nous avons conçu et représenté cet archétype, à l'instar de Morin (op. cit.), comme le cœur d'un processus de différenciation multidimensionnel où des phénomènes organisationnels s'inscrivent dans des dimensions individuelles-collectives, naturelles-artificielles et physiques-cognitives. Dans cette conception systémique, le SFCAT est assimilable à un nœud « computationnel » qui relie quatre sous-systèmes où la prégnance et la qualité des interactions sont déterminantes pour établir sa spécificité et son potentiel de patrimonialisation.

En posant l'hypothèse d'un accroissement des niveaux d'organisation entre des phénomènes physiques $(\mathbf{1} \rightarrow \mathbf{2}-$ du codage génétique à l'écosystème -) et cognitifs (3) $\rightarrow$ (4) - du système cognitif individuel à l'organisation sociale), les SFCAT sont ainsi dépendants et acteurs :

(1) des spécificités du milieu naturel incorporées dans le produit ;

2 de la prégnance d'un système génétique à la fois productif (technique) et social ; 3 d'un système cognitif spécifiquement dédié à la compréhension, à la mémorisation et à la communication entre des sous-systèmes ;
4 de l'environnement social et culturel, vecteur dans la distribution et la transmission mais aussi moteur des évolutions et innovations. C'est dans cet environnement que s'effectuerait «l'épidémiologie des représentations »(Sperber, 1996).

\section{Des savoir-faire fromagers en perspective dynamique}

Ces systèmes pastoraux anciens marquent encore les représentations collectives de l'élevage corse et de ses produits. Nombreuses sont les icônes du berger, fusil à l'épaule, transhumant avec ses chèvres sur de hauts plateaux (sur les étiquettes de fromages produits industriellement par exemple). L'image est active : elle « fait vendre » un fromage réputé par le système technique auquel il se réfère, recherché par une clientèle qui s'attache aux produits de l'élevage fermier ; l'attachement étant fondé ici sur des souvenirs, des récits, des préjugés, des images symboliques.

La question est alors de savoir s'il existe aussi des savoir-faire spécifiques à la fabrication du fromage permettant d'imaginer un ancrage de même nature que celui de l'élevage et donc d'associer dans un même modèle (ou SFACT) le pastoralisme et la fabrication de fromages traditionnels. 
L'identification de ces savoir-faire autour des traitements du lait semble, en fait, plus délicate. L'activité de transformation du lait à la ferme, en tant qu'activité économique régulière, est en effet récente : les priorités accordées à l'élevage, on l'a vu, mais aussi une forte déprise rurale, enfin la présence jusque dans les années 1970 des industriels de Roquefort pour la collecte du lait de brebis ou des affineurs de Calenzana pour l'affinage du fromage de chèvre expliquent le déploiement tardif de cette activité économique.

La fabrication fermière est attestée dans les élevages au XIX ${ }^{\mathrm{e}}$ siècle. Elle s'est réduite peu à peu à quelques rares familles ou s'est concentrée sur des périodes d'estives durant lesquelles les industriels fermaient les laiteries. Jusque dans les années 90 la transformation laitière est aussi dédiée à des « ventes de traite » : une famille réservait un volume de lait juste trait chez un éleveur ; celui-ci livrait sa commande « en blanc », c'est-à-dire un à deux jours après caillage et son acheteur se chargeait de l'affinage. Ces échanges différenciaient bien la séquence de stabilisation du lait (emprésurage, caillage, mise en moule, salage) réalisée rapidement voire brusquement $^{11}$, de la séquence d'affinage (lavage, retournements) faisant appel à la patience, à l'observation, ainsi qu'à la créativité individuelle, déterminante pour l'apparence finale du fromage (chaque vallée peut ainsi reconnaître ses fromages, marqués d'un style).

La tactique de l'éleveur consistait à cailler le lait, rompre la matière puis la mouler rapidement afin de retourner surveiller son troupeau. Le salage et quelques retournements dans un casgile (grotte souterraine pour la conservation du fromage en montagne) permettaient ensuite le transport du fromage

11. Un chef de fabrication industriel nous confiait : "L'agression violente faite par les bergers traditionnels pour casser des caillés si nobles... mais c'est sans doute grâce à ça qu'ils ont inventé le brocciu. »
« en blanc » vers un lieu d'affinage (celui d'un client ou la cuisine d'une épouse). Le goût final importait peu : la phase de préparation était trop distante (dans l'espace et le temps) de l'acte de consommation luimême ; mais l'éleveur savait que les saveurs d'un fromage dépendaient aussi du climat et de l'alimentation du troupeau.

L'affinage donnait une « touche » finale au fromage : seuls ceux qui maîtrisaient à la fois la connaissance des origines du lait et celle des techniques et du style de son affineur pouvaient précisément différencier les fromages entre eux ${ }^{12}$.

\section{Hybridations et distinctions professionnelles}

Avec le retrait des industriels aveyronnais dans les années 70 , les pouvoirs publics se sont engagés à subventionner d'une part la sédentarisation et la mécanisation des élevages jusque là transhumants, et d'autre part les techniques de transformation fermière (aide à l'installation d'ateliers aux normes européennes). La filière fermière qui représente aujourd'hui environ $30 \%$ des volumes de lait pour environ 350 éleveurs, acquiert alors progressivement une réelle place économique. Elle rencontre néanmoins de nouveaux défis à surmonter, que la recherche met en évidence par différents travaux comparatifs :

- Les savoirs anciens, représentatifs d'une tradition et d'une identité avant tout pastorale, sont aujourd'hui diffus ou confondus avec les savoir-faire prônés par l'entreprise Roquefort qui recrutait des éleveurs reconvertis en fromagers. C'est ainsi que s'est développée chez les industriels comme chez certains artisans fermiers, une tomme de Corse, mixte technologique entre un fromage traditionnel du sud de l'île et un modèle de

12. Ces compétences pour différencier - et nommerdifférents fromages en fonction d'une origine du lait et du « style » technique de l'affinage, se perpétuent actuellement, et contribuent à complexifier les débats sur la sélection et la formalisation de savoirfaire spécifiques aux fromages corses. 
tomme standard issue d'innovations industrielles. Un phénomène de percolation s'est opéré, l'hybridation mêlant aujourd'hui savoirs experts des industries laitières et savoirs empiriques des bergers.

- Les outils eux-mêmes de la transformation ont été modifiés : remplacement du bois par l'aluminium ${ }^{13}$ puis par l'inox et le plastique, apparition du froid, de la conservation sous vide - assurée avant par le sel ou la fumée recours à des adjuvants stabilisateurs comme les ferments lactiques. Ces évolutions sont d'immédiates traductions technologiques d'une triple évolution des habitudes alimentaires, des réalités économiques et des normes sanitaires.

- Des jeunes se sont installés après des études universitaires ou comme nouveaux ruraux du continent. Ils défendent des savoir-faire issus de leurs formations auxquels ils ajoutent les effets d'une expérience ou les témoignages locaux de leurs nouveaux réseaux sociaux. - De nouvelles logiques d'action émergent : pour réduire les incertitudes liées à l'environnement et sécuriser les systèmes, la connaissance tacite ou d'expériences du cheptel et du milieu diminue au profit d'artefacts technologiques garantissant les performances techniques de l'élevage et l'efficacité de la transformation laitière.

- Certains éleveurs tendent à concentrer leurs apprentissages sur ces nouveaux dispositifs technologiques en s'appuyant sur des techniciens spécialisés issus des écoles et des laiteries, dotés de savoirs formalisés (guide des bonnes pratiques, manuels agronomiques ou zootechniques).

Les savoir-faire d'élevage sont désormais dissociés des savoir-faire fromagers. Des spécialisations de métier se sont développées et la combinaison des deux activités relève désormais d'un engagement individuel, organisé et construit par des références plus militantes ou idéalistes que fonctionnelles.

13. Certains panneaux routiers indicateurs de direction en aluminium galvanisé ont été reconvertis en planche d'égouttage.
- Avec de plus en plus d'ateliers aux normes européennes et une préoccupation croissante de « réussite hygiéniste » la spécialisation fermière adoptée par les jeunes installés repose sur l'apprentissage de savoirs fermiers nouveaux. Pour obtenir un fromage conforme, « sain, loyal et marchand », ils sont confrontés, au même titre que d'autres artisans, à des technologies directement importées de l'industrie laitière et de ses écoles (ENIL), prescrites par les services sanitaires.

- Dans cette configuration, les fromagers mettent en œuvre des savoir-faire procéduraux et routiniers, formalisés et intériorisés à travers les formations et les suivis techniques réguliers. Ces nouveaux producteurs de fromages mobilisent de moins en moins leurs capacités d'ajustements cognitifs, leur créativité, tant les aléas et les variations techniques sont aujourd'hui anticipés, nivelés et corrigés par des artefacts technologiques introduits tout au long du processus. Les savoir-faire s'exonèrent de plus en plus d'une maîtrise approfondie du milieu naturel et d'un médiateur culturel local : leur acquisition n'est plus dépendante d'une immersion culturelle mais s'est déplacée dans une relation étroite avec un conseiller fromager et des fournisseurs de matériels, porteurs d'un savoir faisant référence à une littérature technique.

- L'observation parallèle de certaines pratiques dites familiales qui matérialisent d'anciens savoir-faire et se traduisent par la mobilisation d'outils généralement proscrits par les normes technicistes, donne cependant quelques repères supplémentaires à ces jeunes éleveurs, des comparatifs à défaut d'être pour eux des références toutes puissantes. C'est cette possibilité qui leur permet de réaliser des hybridations techniques inattendues, revendiquées plus tard comme étant bien elles aussi des traditions techniques typiques d'un savoir-faire fromager, offrant un fromage chaque fois singulier (l'hybridation n'étant pas réalisée de manière identique et homogène d'un individu à l'autre). 


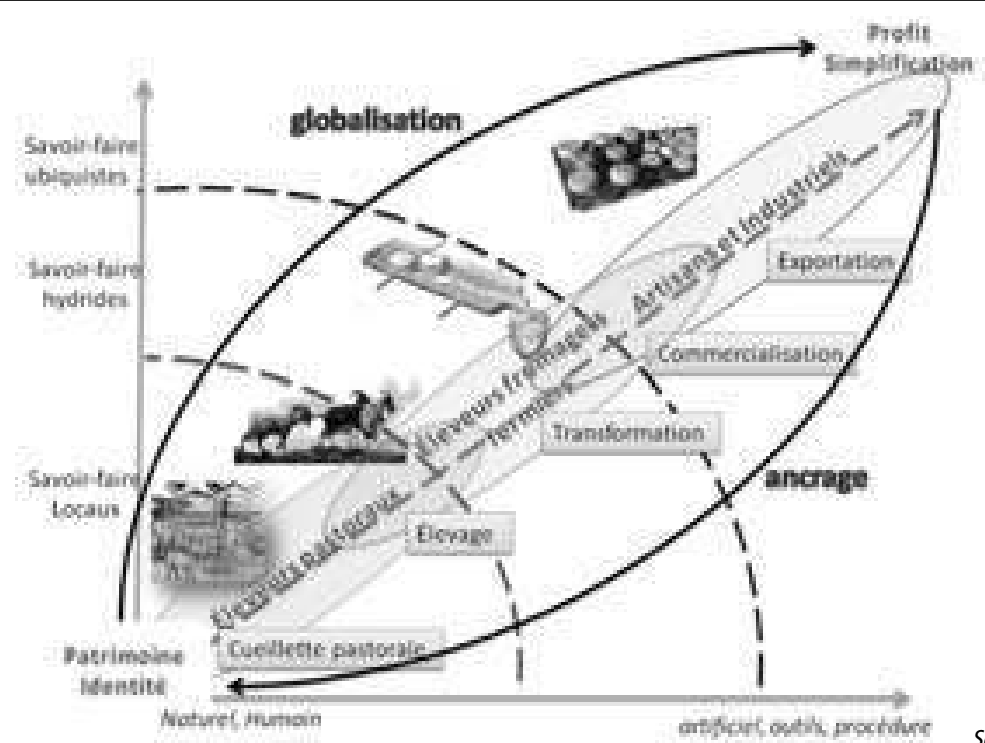

- Enfin ce sont ces constructions sociotechniques, traduites en productions spécifiques, revendiquées comme telles, qui nous permettent d'identifier un SYAL fromager corse ${ }^{14}$.

\section{Hybridations et mondes pour qualifier}

Au terme de ce parcours au sein du SYAL fromager corse, il est possible de représenter (figure 2) ces savoir-faire que la recherche a permis de révéler, agencés le long d'un axe allant de la production à la commercialisation, de l'herbe au fromage en passant par différentes étapes d'élaboration et de transformation. Nous projetons cet axe dans un espace cartésien entre :

- un axe des ordonnées représentant une échelle de généralisation des savoirs depuis les savoirs locaux (traditionnels) spécifiques, ancrés dans un contexte local culturel et écologique jusqu'aux savoirs

14. Si l'on s'en tient aux deux critères du style technique et de l'origine du lait (saisonnière ou micro-territoriale) pour distinguer des fromages, on parlera de plusieurs SYAL fromagers ; mais c'est là l'objet d'un autre débat, scientifique et politique à la fois. les plus génériques et procéduraux ${ }^{15}$ dont l'application peut se déployer indifféremment du contexte local à celui d'une logique économique globale ;

- un axe des abscisses distinguant le substrat cognitif de ces artefacts, soulignant ainsi les temporalités et modalités d'appropriation, de transmission et d'apprentissage que cela induit.

Sur ce graphe, des cercles concentriques (qu'il y a lieu d'imaginer « poreux ») partant de l'origine, figurent d'abord le cœur du système qui décrit en fait le SYAL; le mouvement d'éloignement vers des périphéries ponctuées d'espaces interstitiels signale différentes hybridations. Cette représentation schématique met en évidence un mouvement constant, mû par un double champ de forces :

- d'une part, une tendance centrifuge qui accompagne naturellement l'innovation

15. Ces savoir-faire procéduraux sont enseignés dans les écoles de commerce ou de laiterie, puis déployés avec un faible coût adaptatif, dans une logique de maîtrise technologique du produit. Ils sont nommés « savoir ubiquistes » dans la figure 2 . 
vers une simplification matérielle et cognitive (marché, technologie, procédures) ; - d'autre part, des forces centripètes de réancrage vers le cœur du SYAL où s'expriment les spécificités, les appartenances, les identités.

En convenant de signaler sur ce plan les trois catégories socioprofessionnelles rencontrées, nous visualisons par ailleurs des « zones d'hybridation » de savoir-faire et leurs enjeux. On peut ainsi situer :

- Des savoir-faire pastoraux et leur spécificité dans la production d'un lait à partir d'une race locale et d'un parcours méditerranéen. Cet ensemble de savoirs locaux qualifiés de SFCAT ne dispose pas d'artefact technologique. Il est avant tout dépendant d'une compétence humaine reposant sur des apprentissages au cours de la vie mais aussi sur des efforts pour capter les informations nécessaires au pilotage. Ces savoir-faire sont à la fois spécifiques et adaptatifs selon les circonstances. Mais les éleveurs qui les détiennent sont peu enclins à les revendiquer dans les arènes de certification des produits.

- Des savoir-faire procéduraux portés par les industries et le marketing indispensables pour intégrer une logique économique et conquérir une notoriété ; ils s'appuient sur des artefacts technologiques et des connaissances transposables ; imitables et standardisés ils ne laissent pas la possibilité de défendre la spécificité du fromage.

- Enfin, entre ces deux univers du pastoral et de l'industriel, se construisent des savoirfaire ni tout à fait ancrés à la tradition pastorale ni complètement médiatisés par la technologie, portés par de nouveaux fromagers. En quête d'artefacts techniques pour combler leur déficit cognitif concernant les objets de la nature (troupeau, écosystème) et les humains (langue, rites de communication) ils se concentrent plutôt sur des tâches de transformation et de commercialisation. Ils s'engagent souvent dans des revendications syndicales portant sur leur légitimité et leur ancrage au territoire. Ces fromagers éloignés des savoir-faire pastoraux tout en y faisant référence dans leurs revendications sont aussi porteurs de projets patrimoniaux qui ouvrent une perspective d'objectivation du futur possible de ce SYAL.

Ces trois composantes peuvent être aussi analysées en référence aux mondes des conventions de Boltanski et Thévenot (1991) :

- Les éleveurs traditionnels (garants « civiques » d'un intérêt collectif de maintien du patrimoine culturel ancien) sont peu présents encore dans les démarches de qualification et rarement accessibles audelà d'une sphère "domestique » initiée.

- Les nouveaux fromagers inscrits dans les sphères de «l'inspiration » (innovation sur les produits fermiers) et de "l'opinion» (foires rurales, médias), sont militants d'un nouveau cadre identitaire, fermier, insulaire, qui cherche à rendre possible l'activation, l'expression et la perpétuation de savoir-faire traditionnels.

- Enfin, les industriels, inscrits dans des mondes «marchand» et «industriel » de la fabrication fromagère, qui pour faire reconnaître (bien au-delà des frontières insulaires) les spécificités des produits locaux délaissent toute référence à la complexité des ressources socio-cognitives locales, fondatrices de ces SFACT.

\section{Conclusion \\ Dialectique entre forces centrifuges et centripètes de la patrimonialisation}

La « mise en patrimoine » de ressources naturelles et cognitives est un processus particulièrement présent dans le SYAL fromager corse. Elle prend la forme d'une action collective, encadrée par divers organes et institutions s'attachant à construire tout à la fois un objet-emblème, une ressource économique et politique. 
Ce patrimoine recouvre à la fois la maîtrise d'une génétique adaptée (des cheptels), les modes d'utilisation d'un environnement naturel, des codes cognitifs et relationnels spécifiques, inscrits et transmis dans des logiques normatives locales. Pourtant, son ancrage historique paraît affaibli car difficilement formalisable ou transposable aux nouvelles générations. Les savoir-faire qui s'y rattachent sont d'une part difficiles à identifier et à recueillir, d'autre part ils coévoluent difficilement avec l'environnement technique, culturel et social (Bouche, 2008). Leur transmission passe essentiellement par la perpétuation de pratiques adaptatives dégagées du langage qui permettrait de les formaliser.

Mais dans le processus actuel d'une création patrimoniale institutionnalisée qui serait construite sur les compétences et les identités locales d'un côté et sur les attentes des marchés exogènes de l'autre, la tradition orale nous apparaît comme un nouvel « activateur de changement » : réhabilitée dans les collectifs consultés, elle forme en effet la trame des liens que tentent désormais de tisser les nouveaux éleveurs avec les anciens. Mais ces derniers ne se projettent guère dans un devenir collectif. Porteurs de l'identité pastorale, ils sont pourtant peu actifs pour revendiquer la reconnaissance ou la protection de leur système pastoral et fromager de référence. Dans ce contexte, les nouveaux fromagers qui s'appuient d'abord sur les guides de pratiques normalisées tout en recherchant des liens avec les « anciens », se portent finalement candidats pour construire un processus d'ancrage de leurs savoir-faire se dégageant des contraintes du territoire trop étroitement associé aux savoir-faire de ces « anciens ». Forts d'une conscience identitaire qui les mobilise pour la construction politique d'un patrimoine qu'ils réinventent peut être, ce sont eux qui constituent sans doute une force idéologique et professionnelle légitime pour porter un projet collectif et définir les nouvelles spécificités du SYAL fromager corse.
Le devenir des SFCAT fromagers corses ne peut pourtant pas se contenter de ce seul acteur. Il repose nécessairement sur la capacité de tous (organismes d'appui et acteurs de la filière fromagère) à promouvoir des coordinations innovantes entre les trois composantes ou « mondes », qui cohabitent sans forcément communiquer sur l'espace insulaire corse. Sans prendre en compte ces coordinations locales nécessaires, le risque est grand de construire un dispositif de «normation $»^{16}$ (Pestre, 2008) permettant juste d'inventorier et de transformer des savoirs individuels épars en savoirs instrumentaux et incrémentaux pour une action collective qui s'inscrirait seulement dans une perspective marchande. Or, l'enjeu de renforcement des SYAL n'est pas d'orienter les logiques productives localisées vers les marchés mais bien de construire des articulations significatives localement, entre territoires, savoir-faire identitaires, productions et transmissions (les marchés n'étant qu'une voie pour faire connaître et transmettre des produits à fort ancrage territorial) qui font les patrimoines locaux. Pour passer d'un « être ensemble » à un « agir ensemble » de l'action collective telle que décrite par Livet et Thévenot (1994) et pour l'envisager dans une perspective de développement durable avec ses trois composantes (économique, écologique et sociale), un SYAL ne peut se raisonner qu'au niveau d'une interprofession admettant l'apprentissage nécessaire d'une position médiane entre, d'un côté, les forces centrifuges du progrès et de la mondialisation, et de l'autre, les forces centripètes de l'ancrage.

16. Le terme « normation » est directement emprunté à D. Pestre pour désigner les mises aux normes et leurs effets de fixation. 


\section{RÉFÉRENCES BIBLIOGRAPHIQUES}

Bérard L., Marchenay P. (2004). Les produits de terroir entre cultures et règlements. Paris, CNRS édition, 225 p.

Bérard L., Marchenay P. (2007). Produits de terroir. Comprendre et agir. CNRS, UMR Éco-Anthropologie et Ethnologie, $61 \mathrm{p}$.

Boltanski L., Thévenot L. (1991). De la Justification. Les économies de la grandeur. Paris, Gallimard, 224 p.

Bouche R., Bordeaux C., Aragni Chj. (2009). Ancrage territorial de savoir-faire collectifs au sein d'un Syal : cas des fromages de Corse. In Muchnick J. (dir.), « Le temps des SYAL », édition Quae (sous presse), $14 \mathrm{p}$.

Bouche R. (2008). Mediterranean Pastorality: Sustainable know-how in search of development. In Bouche, Casabianca (dir.), actes du $\mathrm{X}^{\text {th }}$ EAAP Mediterranean Symposium Corte "New trends for innovation in the Mediterranean animal production", 6-8 novembre, http://www.corte.inra.fr/symp-med, 12 p.

Chevallier D., Chiva I. (1991). L'introuvable objet de la transmission. In Chevallier D. (dir.), « Savoir-faire et pouvoir transmettre. Transmission et apprentissage des savoir-faire et des techniques », Paris, édition MSH, p. 1-11.

Deforge Y. (1991). La transmission et la préservation des savoir-faire et les enseignements techniques. In Chevallier D. (dir.), «Savoir-faire et pouvoir transmettre. Transmission et apprentissage des savoir-faire et des techniques », Paris, édition MSH, p. 197-216.

Détienne M., Vernant J.-P. (1974). Les Ruses de l'intelligence. La métis des grecs. Paris, Flammarion, 320 p.

Guillet Escuret G. (2003). Les techniques, entre tradition et intention. Techniques et Cultures, $\mathrm{n}^{\circ} 42$, p. 97-109.
Latour B. (1993). Petites leçons de sociologie des sciences. Paris, La Découverte, 251 p.

Latour B., Lemonnier P. (1994). Introduction. In Latour B., Lemonnier P. (dir.), "De la préhistoire aux missiles balistiques, l'intelligence sociale des techniques », Paris, La Découverte, p. 9-24.

Leroi-Gourhan A. (1965). Le geste et la parole. Sciences d'aujourd'hui, collection dirigé par George A., Paris, Albin Michel, 285 p.

Livet P., Thevenot L. (1994). Les catégories de l'action collective. In Orlean A. (dir.), «Analyse économique des conventions », Paris, PUF, p. 139-168.

Martinelli B. (1993). Sens de la tendance technique. Techniques et Cultures, $\mathrm{n}^{\circ} 21$, p. 1-26.

Martinelli B. (2005). Style, technique et esthétique en anthropologie. In Martinelli B. (dir.), "L'interrogation du style. Anthropologie, technique et esthétique », Aix en Provence, Université de Provence, p. 19-48.

Moity-Maïzi P., Muchnik J. (2005). Circulation et construction de savoir-faire : questions pour une anthropologie des systèmes alimentaires localisées. Industries Alimentaires et Agricoles, $\mathrm{n}^{\circ} 9$, septembre, p. 16-26.

Moity-Maïzi P., Paquin D. (dir.) (2007). Laits, fromages, Corse. Quelles identités valoriser? DVD $26 \mathrm{mn}$, produit par l'Institut des Régions Chaudes, l'UMR Innovation, le GIS SYAL et l'INRA/LRDE de Corte.

Moity-Maïzi P. (2009). L'ancrage territorial mis en question. In Muchnick J. (dir.), "Le temps des SYAL », Paris, Quae, (sous presse).

Morin E. (1973). Le paradigme perdu : la nature humaine. Paris, éditions du Seuil, $246 \mathrm{p}$. 
Muchnik J., Sainte Marie (de) C. (2010). Sigaut F. (2006). Le savoir des couteaux. In Introduction générale. In Muchnik J., Sainte Marie (de) C. (coord.), « Le temps D'Onofrio S., Joulian F. (dir.), « Dire le savoir-faire », Paris, Cahiers d'anthropodes SYAL, Techniques, vivres et territoires », Versailles, Quae, coll. Update, p. 13-29.

Pestre D. (2008). Préface. In De S. Topçu C. Cuny K. Serrano-Velarde (dir.), « Savoirs en débat. Perspectives franco-allemandes », Paris, L'Harmattan, coll. Sciences et Sociétés, p. 11-16.

Ravis-Giordanni G. (1983). Bergers corses, les communautés villageoises du Niolu. Edisud, 505 p. logie sociale 01, L'Herne, p. 133-139. Stehr N. (2000). Le savoir en tant que pouvoir d'action. Sociologie et sociétés, vol. $32, \mathrm{n}^{\circ} 1$, p. 157-170.

Sperber D. (1996). La contagion des idées. Paris, Odile Jacob, 243 p.

Theureau J. 1992, Le cours d'action, analyse sémio-logique : essai d'une anthropologie cognitive située. Berne, Peter Lang, 339 p. Vermersch P. (2010). L'entretien d'explicitation, Esf, 220 p.

Schwint D. (2002/2). Savoir artisan de fabriWagensberg J. (1997). L'âme de la méduse : cation et détournements du temps. Sociétés $\mathrm{n}^{\circ} 76$. idées sur la complexité du monde. Paris, Seuil, 169 p. 